\title{
Perfil pneumofuncional de aplicadores de agrotóxicos no nordeste do Pará - Brasil
}

\author{
Pneumofunctional profile of agrochemical applicators in northeast of Pará - Brazil
}

Samires Avelino de Souza França', Paulo Eduardo Santos Ávila², Valéria Marques Ferreira Normando ${ }^{3}$

Resumo Objetivo: Avaliar a função pulmonar de trabalhadores rurais expostos a agrotóxicos no nordeste do Pará. Método: Estudo observacional, transversal, analítico, realizado no município de Bujarú (Pará- Brasil). Os voluntários (n=26) foram submetidos à uma avaliação geral e pneumológica. Peso e altura foram obtidos com balança analógica Hidromel; prerssão arterial mensurada com esfigmomanômetro e estetoscópio Premium; função pulmonar avaliada com Espirômetro MicroQuark - COSMED, baseada nas recomendações do I Consenso Brasileiro de Espirometria: indivíduo relaxado, sentado, com a coluna ereta e apoio nas costas, uso de clipe nasal e bocais descartáveis, sendo executadas três expirações máximas sustentadas para escolha das curvas de volume-tempo e fluxo-volume mais homogêneas e com melhores valores de CVF visualizados no software PTF Suite. Para avaliar as variações sofridas pela espirometria utilizou-se o teste de Wilcoxon e a associação entre o perfil pneumofuncional e os fatores de risco foi analisada pelo teste $t$ de Student e Mann-Whitnay. Adotou-se $p<0,05$ para significância estatística e a análise foi realizada pelo software BioEstat 5.0. Resultados: Houve reduções, abaixo do previsto, de todas as variáveis espirométricas entre os trabalhadores, destacando as reduções médias: de $-1,79 \mathrm{~L} / \mathrm{seg}(\mathrm{p}<0,0001)$ para o PFE; $-0,67 \mathrm{~L}(\mathrm{p}<0,0001)$ para CVL; $-0,31 \mathrm{~L}$ $(\mathrm{p}=0,0026)$ para CVF; -0,28L ( $\mathrm{p}=0,0032)$ para VEF1; -0,21\% ( $<<0,0001)$ para VEF1/CVF. Cerca de 11,5\% dos indivíduos apresentaram distúrbio restritivo de leve a moderado e 46,2\% mostraram padrões sugestivos ao desenvolvimento de distúrbios ventilatórios mistos. Conclusão: Sugere-se a predisposição ao desenvolvimento de distúrbios restritivos e mistos entre estes trabalhadores.

Palavras-chave: espirometria; agroquímicos; exposição ocupacional; saúde da população rural.

Summary Purpose: To evaluate the pulmonary function of rural workers exposed to pesticides in the northeast of Pará. Methods: An observational, cross-sectional, analytical study Developed in the municipality of Bujaru, Para State -Brazil. The volunteers $(n=26)$ were underwent a general and pneumological evaluation. Weight and height were obtained with Hydromel's analogue balance; prersion measured with Premium's sphygmomanometer and stethoscope. Pulmonary function evaluated with MicroQuark Sprinkler - COSMED, based on the recommendations of the I Brazilian Consensus of Spirometry: relaxed, sitting individual with spine up and back support, use of nasal clip and disposable nozzles, performing three maximum sustained expirations to choose the more homogeneous volume-time and volume-volume curves and better CVF values visualized in the PTF Suite software. Wilcoxon's test was used to evaluate the variations suffered by spirometry and the association between the pneumofunctional profile and the risk factors was analyzed by Student's $t$ test and Mann-Whitnay test. We adopted $\mathrm{p}<0.05$ for statistical significance and the analysis was performed by BioEstat 5.0 software. Results: There were decreases, below the predicted, of all spirometric variables among workers, highlighting the mean reductions: $-1,79 \mathrm{~L} / \mathrm{sec}(\mathrm{p}<0,0001)$ for PEF; $-0,67 \mathrm{~L}$ $(\mathrm{p}<0,0001)$ for LVC; $-0,31 \mathrm{~L}(\mathrm{p}=0,0026)$ for FVC; $-0,28 \mathrm{~L}(\mathrm{p}=0,0032)$ for FEV1; $-0,21 \%(\mathrm{p}<0,0001)$ for FEV1 / FVC. About 11,5\% of the individuals had a mild to moderate restrictive disorder and $46.2 \%$ showed patterns suggestive of the development of mixed ventilatory disorders. Conclusion: We suggest the predisposition to the development of restrictive and mixed disorders among these workers.

Keywords: spirometry; agrochemicals; occupational exposure; rural health.

'Universidade Federal do Pará - UFPA, Belém, Pará, Brasil

¿Universidade da Amazônia - UNAMA, Belém, Pará, Brasil

3Universidade do Estado do Pará - UEPA, Belém, Pará, Brasil

Fonte de financiamento: Nenhuma.

Conflito de interesses: Os autores declaram não haver conflitos de interesse.

Recebido: Julho 24, 2018

Aceito: Agosto 31, 2018

Trabalho realizado na Universidade da Amazônia - UNAMA, Belém, PA, Brasil. 


\section{Introdução}

A produção agrícola contribui subtancialmente para impulsionar a economia brasileira, estando o Brasil no ranking dos maiores consumidores globais de agrotóxicos ${ }^{1}$. A exposição ocupacional à pesticidas acarreta diversos prejuízos à saúde respiratória dos indivíduos. Dentre as principais queixas associadas à inalação de defensivos químicos detacam-se: chiado e aperto no peito, falta de ar e tosse com expectoração durante o trabalho².

Nos países do terceiro mundo, como o Brasil, o uso de defensivos químicos nos setores agrícolas tem se tornado cada vez mais freqüente. Inúmeras atividades agrícolas envolvem exposição a elevados níveis de poeira, gases tóxicos e defensivos químicos. Os agrotóxicos são substâncias utilizadas na agricultura para manutenção da colheita, controle de insetos e outras pragas nocivas. Contudo, seu uso incorreto e indiscriminado pode oferecer riscos à saúde. O desenvolvimento, as características e a gravidade dos distúrbios respiratórios são proporcionais à concentração do agente químico, o tempo de exposição e a suscetibilidade de cada trabalhador ${ }^{3,4}$.

As respostas fisiológicas humanas à exposição aos agrotóxicos variam e podem ser potencializadas de acordo com alguns fatores individuais. O tabagismo, etilismo, o nível de escolaridade, o estado nutricional de cada indivíduo, a não utilização dos equipamentos de proteção individual; alimentar-se durante a aplicação dos defensivos químicos; higiene pessoal inadequada; o armazenamento do produto químico na própria residência; e as condições climáticas podem potencializar os efeitos nocivos do trabalho com agrotóxicos ${ }^{1,4,5}$.

A espirometria é uma ferramenta bastante utilizada na prática clínica, auxiliando e subsidiando o diagnóstico de doenças pulmonares e a identificação de riscos para o desenvolvimento de disfunções ventilatórias em diversas populações. Estudos transversais, caso-controle e coortes prospectivas têm demonstrado alterações da função pulmonar entre trabalhadores expostos à inalação de agrotóxicos ${ }^{6-10}$. O objetivo desse estudo foi avaliar a função pulmonar de trabalhadores rurais expostos à agrotóxicos, correlacionando-a ainda alguns fatores de risco (sintomatologia, tabagismo, utilização ou não de equipamentos de proteção individual e o tempo de exposição aos agroquímicos).

\section{Metodologia}

Trata-se de um estudo observacional, transversal; desenvolvido no município de Bujarú - Pará - Brasil. Foi visitada uma propriedade rural onde se cultivava a pimenta-do-reino e onde trabalhavam aplicadores de agrotóxicos. Os agrotóxicos utilizados pelos agricultores eram os herbicidas Gramoxone e Roundup e os inseticidas Sultox e Cipermetrina.

Este estudo foi aprovado pelo Comitê de Ética em Pesquisa (CEP) Envolvendo Seres Humanos da Universidade da Amazônia, sob o parecer 348193/10). Os voluntários desta pesquisa assinaram um Termo de Consentimento Livre e Esclarecido (TCLE), declarando total ciência em relação ao objetivo da pesquisa, além dos riscos e dos benefícios, bem como da sua participação voluntária.

A amostragem se constituiu de vinte e seis (26) trabalhadores rurais do sexo masculino, com faixa etária compreendida entre 20 e 65 anos e que trabalhavam com a aplicação de defensivos químicos. Foram excluídos da pesquisa os trabalhadores que não estivessem envolvidos na aplicação de agrotóxicos durante o cultivo da pimenta; que já possuíssem diagnóstico clínico fechado de doenças pulmonares ou demais patologias associada; voluntários com déficits cognitivos que limitassem a execução da prova espirométrica; bem como menores de 18 anos e aqueles que se recusaram a assinar o TCLE.

Os 26 participantes foram subdivididos de acordo com alguns fatores de risco: tabagismo, (tabagistas [n=17] e não tabagistas [n=9]); uso de equipamentos de proteção individual (usuários de EPI [n= 8] e não usuários de EPI [ $n=18]$ ), sintomatologia (indivíduos com sintomas respiratórios [ $n=9]$ e indivíduos sem sintomas [n=17]) e de acordo com tempo de exposição a agrotóxicos (trabalhadores com menos de 10 anos de exposição [n=16] e trabalhadores com mais de 10 anos de exposição [n=10]).

Este estudo incluiu uma ficha avaliativa geral, que levou em consideração os dados de identificação, o peso, a altura e a idade de cada voluntário. Foi realizada ainda uma avaliação pneumológica de cada trabalhador, considerando dados gerais, o registro de sintomatologia respiratória (queixas), exame físico, ausculta pulmonar, pesquisa de sinais vitais, além de um teste espirométrico.

Os dados da espirometria foram obtidos através do Espirômetro MicroQuark da marca COSMED associado a um software de computador (PTF Suite) que permitiu a visualização das variáveis espirométricas. 
Os trabalhadores também responderam a um questionário contendo perguntas abertas e fechadas referentes a: identificação do voluntário, seus hábitos de vida, tipo de ocupação, queixas, sintomas evidenciados após a aplicação dos agrotóxicos e progressão desses sintomas, utilização de EPI e jornada de trabalho.

Os dados da avaliação clínica geral e pneumológica foram obtidos em um primeiro momento e de forma individualizada. O peso e a altura dos indivíduos foram mensurados através de uma balança analógica da marca Hidromel. A FC foi quantificada através da palpação do pulso radial no intervalo de um minuto e a FR obtida através da visualização dos movimentos torácicos durante um minuto. A PAS foi mensurada através do método auscultatório indireto de Korotkoff por meio de um esfigmomanômetro e estetoscópio, ambos da marca Premium, tendo como referência a artéria braquial esquerda. A ausculta pulmonar foi realizada utilizando o estetoscópio supracitado e levou em consideração pontos auscultatórios anteriores, posteriores e laterais do tórax. A definição do tipo de tórax e do padrão ventilatório adotado pelo trabalhador foi feita através de inspeção. Após esta avaliação, os trabalhadores responderam, ainda, ao questionário específico já mencionado.

O segundo momento da pesquisa equivaleu à prova espirométrica. Os testes foram realizados de acordo com as orientações descritas pelo I Consenso Brasileiro de Espirometria ${ }^{11}$. Cada trabalhador foi devidamente instruído sobre os procedimentos necessários para a realização da prova. Foram executadas três tentativas, dentre as quais foi selecionada aquela cujo resultado apresentou as curvas de fluxo-volume e volume-tempo mais lineares e homogêneas, bem como melhores valores de CVF. O teste foi realizado com o paciente relaxado, sentado, com a coluna ereta e apoio nas costas e associado ao uso de um clipe nasal e bocais descartáveis para biossegurança dos voluntários.

Os valores espirométricos coletados foram catalogados e organizados em uma planilha eletrônica, elaborada através do programa Microsoft Office Excel 2007. Posteriormente, os dados obtidos foram comparados aos valores de normalidade preditos considerando idade, sexo e altura de cada indivíduo ${ }^{12}$.

Para avaliar as variações sofridas pela espirometria, no tocante a diferença entre o observado e o esperado foi aplicado o teste de Wilcoxon para amostra pareadas devido a amostra não ser compatível com a curva de Gauss. E para avaliar a associação entre o perfil pneumofuncional e os fatores de risco, foi aplicado o teste t de Student e, quando a amostra apresentou heterocedasticidade, foi aplicado o teste U de Mann-Whitnay. Foi previamente fixado o $\mathrm{p}<0.05$ para significância estatística. Todo o processamento estatístico foi suportado pelo software BioEstat versão $5.0^{12}$.

\section{Resultados}

O percentual de indivíduos ( $\mathrm{n}=26$ ) com redução dos valores espirométricos abaixo do predito pode ser observado no Gráfico 1.

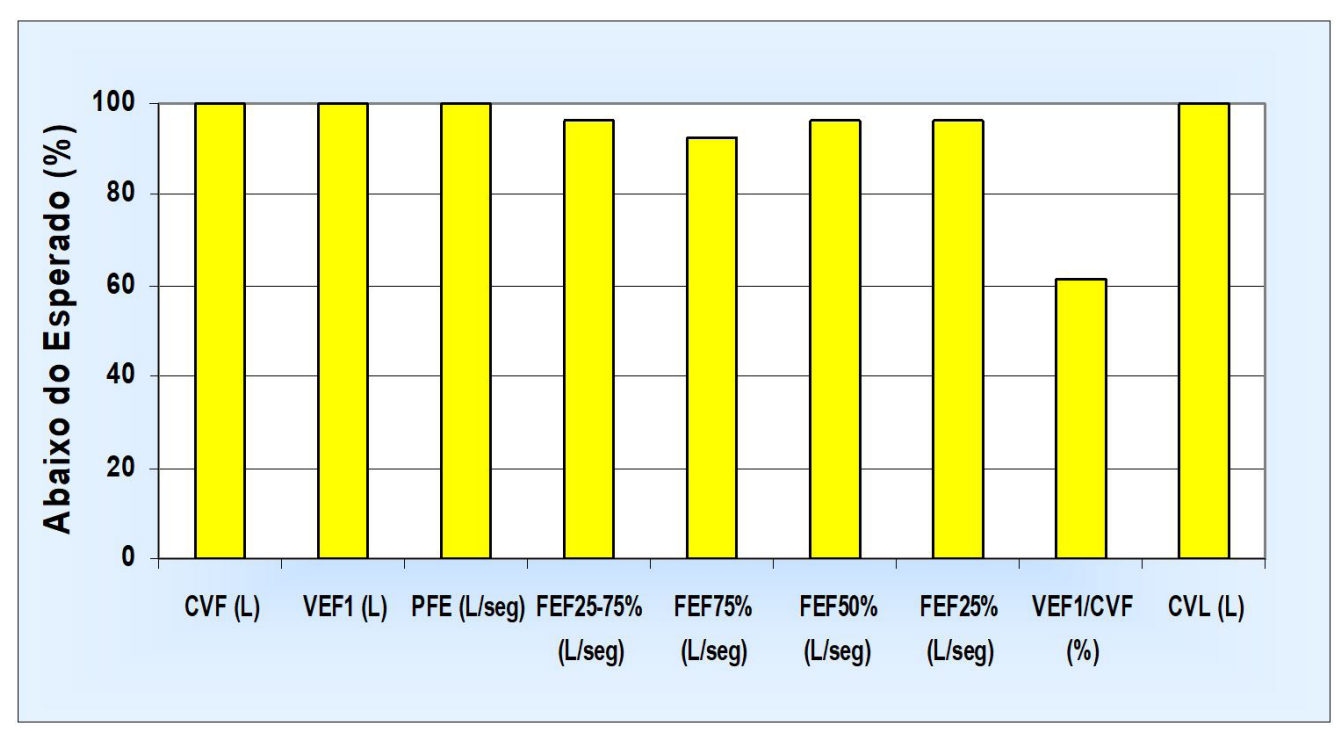

Gráfico 1. Percentual de indivíduos com valores espirométricos abaixo do predito ( $\mathrm{n}=26$ trabalhadores do cultivo de pimenta-do-reino no município de Bujaru - Pará). Fonte: Protocolo da Pesquisa, 2010. 
Os achados estatísticos não demonstraram diferença significativa entre os declínios espirométricos apresentados pelos indivíduos com sintomatologia $(n=9)$, quando comparados a trabalhadores sem sintomas $(\mathrm{n}=17)(\mathrm{p}>0,05)-$ Tabela 1.

A Tabela 2 revela a influência da utilização de EPI sobre a função respiratória, com destaque ao TFEF $(\mathrm{p}<0,05)$.

A tabela 3 aponta a influência do tabagismo na função pulmonar dos trabalhadores expostos a agrotóxicos, com destaque a CVF $(\mathrm{p}<0,05)$.

A Tabela 4 demonstra a influência do tempo de exposição a agrotóxicos sobre a função pulmonar dos trabalhadores, considerando até 10 anos $(n=16)$ e mais de 10 anos de exposição $(n=10)$. Merece destaque FEF25\% $(p<0,050)$

Tabela 1. Avaliação da diferença entre Observado e Esperado conforme a presença de sintoma(s) em uma amostra de $n=26$ trabalhadores do cultivo de pimenta-do-reino no município de Bujaru - Pará

\begin{tabular}{|c|c|c|c|c|c|}
\hline & \multicolumn{2}{|c|}{ Com Sintoma(s) $(n=9)$} & \multicolumn{2}{|c|}{ Sem Sintoma $(n=17)$} & \multirow[b]{2}{*}{ p-valor } \\
\hline & $\begin{array}{l}\text { Média das } \\
\text { diferenças }\end{array}$ & Desvio Padrão & $\begin{array}{l}\text { Média das } \\
\text { diferenças }\end{array}$ & Desvio Padrão & \\
\hline CVF (L) & $-0,24$ & 0,46 & $-0,37$ & 0,47 & 0,5197 \\
\hline VEF1 (L) & $-0,22$ & 0,44 & $-0,31$ & 0,44 & 0,5997 \\
\hline PFE (L/seg) & $-1,78$ & 1,10 & $-1,80$ & 1,16 & 0,9811 \\
\hline FEF25-75\% (L/seg) & $-0,37$ & 0,63 & $-0,53$ & 0,80 & 0,5757 \\
\hline FEF75\% (L/seg) & $-0,97$ & 1,14 & $-1,14$ & 1,46 & 0,7545 \\
\hline FEF50\% (L/seg) & $-0,44$ & 0,77 & $-0,61$ & 0,97 & 0,6351 \\
\hline FEF25\% (L/seg) & $-0,22$ & 0,57 & $-0,21$ & 0,54 & 0,9545 \\
\hline TFEF (s) absoluto & 5,52 & 2,44 & 6,01 & 2,76 & 0,6443 \\
\hline VEF1/CVF (\%) & 0,66 & 3,52 & $-0,13$ & 3,67 & 0,5829 \\
\hline CVL (L) & $-0,57$ & 0,65 & $-0,74$ & 0,67 & 0,5245 \\
\hline
\end{tabular}

Teste aplicado: t de Student. Fonte: Protocolo da pesquisa, 2010.

Tabela 2. Avaliação da diferença entre Observado e Esperado conforme o uso de EPI em uma amostra de $n=26$ trabalhadores do cultivo de pimenta-do-reino no município de Bujaru - Pará

\begin{tabular}{|c|c|c|c|c|c|}
\hline & \multicolumn{2}{|c|}{ Com EPI $(n=8)$} & \multicolumn{2}{|c|}{ Sem EPI $(n=18)$} & \multirow[b]{2}{*}{ p-valor } \\
\hline & $\begin{array}{l}\text { Média das } \\
\text { diferenças }\end{array}$ & Desvio Padrão & $\begin{array}{l}\text { Média das } \\
\text { diferenças }\end{array}$ & Desvio Padrão & \\
\hline CVF (L) & $-0,22$ & 0,40 & $-0,36$ & 0,49 & 0,4978 \\
\hline VEF1 (L) & $-0,18$ & 0,40 & $-0,32$ & 0,45 & 0,4423 \\
\hline PFE (L/seg) & $-2,04$ & 0,77 & $-1,68$ & 1,24 & 0,4590 \\
\hline FEF25-75\% (L/seg) & $-0,49$ & 0,88 & $-0,45$ & 0,68 & 0,8868 \\
\hline FEF75\% (L/seg) & $-1,21$ & 0,76 & $-1,00$ & 1,51 & 0,2704 \\
\hline FEF50\% (L/seg) & $-0,48$ & 0,65 & $-0,57$ & 0,98 & 0,8017 \\
\hline FEF25\% (L/seg) & 0,06 & 0,61 & $-0,34$ & 0,47 & 0,0831 \\
\hline TFEF (s) absoluto & 3,44 & 0,99 & 6,85 & 2,38 & $0,0003^{*}$ \\
\hline VEF1/CVF (\%) & 0,01 & 0,79 & 0,08 & 0,85 & 0,4094 \\
\hline CVL (L) & 0,25 & 1,11 & 0,58 & 1,84 & 0,7010 \\
\hline
\end{tabular}

${ }^{*}$ Teste t de Student. Fonte: Protocolo da pesquisa, 2010. 
Tabela 3. Avaliação da diferença entre Observado e Esperado conforme o Tabagismo em uma amostra de $n=26$ trabalhadores do cultivo de pimenta-do-reino no município de Bujaru - Pará

\begin{tabular}{|c|c|c|c|c|c|}
\hline & \multicolumn{2}{|c|}{ Fumante $(n=17)$} & \multicolumn{2}{|c|}{ Não Fumante $(n=9)$} & \multirow[b]{2}{*}{ p-valor } \\
\hline & $\begin{array}{l}\text { Média das } \\
\text { diferenças }\end{array}$ & Desvio Padrão & $\begin{array}{l}\text { Média das } \\
\text { diferenças }\end{array}$ & Desvio Padrão & \\
\hline CVF (L) & $-0,50$ & 0,38 & 0,00 & 0,33 & $0,0037^{*}$ \\
\hline VEF1 (L) & $-0,39$ & 0,40 & $-0,07$ & 0,44 & 0,073 \\
\hline PFE (L/seg) & $-1,63$ & 1,28 & $-2,09$ & 0,63 & 0,2334 \\
\hline FEF25-75\% (L/seg) & $-0,53$ & 0,68 & $-0,33$ & 0,83 & 0,5008 \\
\hline FEF75\% (L/seg) & $-1,04$ & 1,57 & $-1,12$ & 0,71 & 0,4042 \\
\hline FEF50\% (L/seg) & $-0,77$ & 0,89 & $-0,10$ & 0,71 & 0,0617 \\
\hline FEF25\% (L/seg) & $-0,31$ & 0,55 & $-0,04$ & 0,49 & 0,2243 \\
\hline TFEF (s) absoluto & 5,98 & 2,52 & 5,47 & 2,83 & 0,6427 \\
\hline VEF1/CVF (\%) & $-0,15$ & 3,55 & 0,88 & 3,69 & 0,4833 \\
\hline CVL $(\mathrm{L})$ & $-0,84$ & 0,63 & $-0,36$ & 0,60 & 0,0711 \\
\hline
\end{tabular}

${ }^{*}$ Teste t de Student. Fonte: Protocolo da pesquisa, 2010.

Tabela 4. Avaliação da diferença entre Observado e Esperado conforme o tempo de exposição em uma amostra de n=26 trabalhadores do cultivo de pimenta-do-reino no município de Bujaru - Pará

\begin{tabular}{|c|c|c|c|c|c|}
\hline & \multicolumn{2}{|c|}{ Até 10 anos $(n=16)$} & \multicolumn{2}{|c|}{ Mais que 10 anos $(n=10)$} & \multirow[b]{2}{*}{ p-valor } \\
\hline & $\begin{array}{l}\text { Média das } \\
\text { diferenças }\end{array}$ & Desvio Padrão & $\begin{array}{l}\text { Média das } \\
\text { diferenças }\end{array}$ & Desvio Padrão & \\
\hline CVF (L) & $-0,22$ & 0,37 & $-0,46$ & 0,57 & 0,2113 \\
\hline VEF1 (L) & $-0,18$ & 0,39 & $-0,44$ & 0,48 & 0,0844 \\
\hline PFE (L/seg) & $-1,67$ & 1,02 & $-1,98$ & 1,27 & 0,8080 \\
\hline FEF25-75\% (L/seg) & $-0,37$ & 0,81 & $-0,61$ & 0,58 & 0,2968 \\
\hline FEF75\% (L/seg) & $-0,07$ & 0,84 & $-0,07$ & 1,01 & 0,6248 \\
\hline FEF50\% (L/seg) & $-0,37$ & 0,88 & $-0,82$ & 0,85 & 0,2136 \\
\hline FEF25\% (L/seg) & $-0,03$ & 0,57 & $-0,51$ & 0,34 & $0,0247^{*}$ \\
\hline TFEF (s) absoluto & 5,36 & 2,63 & 6,51 & 2,49 & 0,2779 \\
\hline VEF1/CVF (\%) & 0,65 & 3,86 & $-0,51$ & 3,07 & 0,4297 \\
\hline CVL (L) & $-0,52$ & 0,47 & $-0,92$ & 0,83 & 0,1314 \\
\hline
\end{tabular}

*Teste $t$ de Student. Fonte: Protocolo da pesquisa, 2010.

\section{Discussão}

A espirometria revelou que $11,5 \%$ dos indivíduos apresentavam distúrbio restritivo de leve a moderado consolidado. Entretanto, os achados permitem concluir que dentre os indivíduos com espirometria normal, 46,2\% apresentaram características sugestivas ao desenvolvimento de distúrbios ventilatórios mistos, ou seja, redução de CVF, VEF1, CVL, com diminuição concomitante da relação VEF1/CVF (Gráfico 1).

Cerca de $42,3 \%$ dos trabalhadores apresentaram redução de CVF, CVL e VEF1, mantendo valores da relação VEF1/CVF dentro da faixa de normalidade ou superiores. Diante disto, é possível verificar a predisposição destes indivíduos ao desenvolvimento de distúrbio restritivo da ventilação, mas não se pode confirmá-la, já que a espirometria básica não fornece os valores referentes ao volume residual (VR).

O aplicador de agrotóxico bujaruense, de maneira geral, apresentou redução significativa de todas as variáveis vislumbradas pela espirometria, ao analisarem-se valores preditos e obtidos para cada 
trabalhador (Gráfico 1). Merece destaque o PFE, que demonstrou uma redução média de $-1.79 \mathrm{~L} / \mathrm{seg} \mathrm{e}$ um p-valor $<0.0001$, representando, dessa forma, a tendência de o trabalhador rural apresentar reduções abaixo do previsto para essa variável. A CVL, CVF, o VEF1 e a relação VEF1/CVF também se encontraram consideravelmente reduzidas nesses agricultores; com uma média de perdas de $-0,67 \mathrm{~L}$ ( $\mathrm{p}$-valor $<0.0001$ ), $-0,31 \mathrm{~L}(\mathrm{p}$-valor $=0.0026) ;-0,28 \mathrm{~L}(\mathrm{p}$-valor $=0.0032)$ e $-0,21 \%$ (p-valor $<0.0001)$ respectivamente.

Diversos pesquisadores encontraram redução significativa da CVF ${ }^{13,14}$, VEF1 ${ }^{7-10}, \operatorname{VEF} 1 / \mathrm{CVF}^{7,10,14,15} \mathrm{e}$ FEF $25-75 \%$ \%,14,16. Estas divergências encontradas na literatura sustentam a teoria de que os efeitos nocivos dos agroquímicos têm relação direta com o tipo de agrotóxico utilizado, suas concentrações e com as técnicas de manuseio que diferem em várias regiões. A maior predisposição aos distúrbios mistos, observada nos indivíduos deste estudo, pode estar relacionada com a heterogeneidade de agrotóxicos manuseados pela amostra.

Sibilos, dispnéia, tosse crônica e asma são sintomas bastante evidentes em aplicadores de agrotóxicos e estão, geralmente, relacionados ao quadro de obstrução das vias aéreas. Sua ocorrência foi acompanhada pela redução dos valores de PFE e FEF\% ${ }^{17-19}$. Entretanto, não houve relação, estatisticamente significante, entre a presença de sintomas e a conseqüente redução dessas variáveis. Pode-se inferir que os declínios espirométricos observados nesse estudo não se refletiram na exacerbação de sintomas, talvez por ainda não constituírem redução considerável da função pulmonar.

Quanto aos trabalhadores que utilizam EPI (31\%) e os desprovidos desses equipamentos (69\%), não houve diferença estatística entre as reduções espirométricas sofridas por esses indivíduos. Os aplicadores que fizeram uso de luvas, máscara simples, macacão e botas não tiveram sua saúde respiratória significativamente respaldada pelo uso exclusivo desses equipamentos, ao contrário: esses aplicadores apresentaram declínios bastante semelhantes aos dos trabalhadores desprovidos de proteção (Tabela 2). Esse achado justifica a necessidade de investimentos em infra-estrutura do trabalho, para que as boas condições do meio ocupacional possam potencializar os efeitos benéficos do uso regular de EPI.

Programas de engenharia já tem sido pensados e estimulados em países como os Estados Unidos, com a formulação de uma lei de segurança ocupacional, a qual determina que os empregadores devem fornecer ambientes ocupacionais devidamente equipados e que garantam segurança no trabalho. Medidas como esta, de alto valor agregado, somadas a utilização dos EPI têm reduzido o adoecimento e as lesões em âmbito ocupacional ${ }^{20}$.

Em países em desenvolvimento, como o Brasil não é incomum que o uso de EPI seja negligenciado pelo trabalhador, o qual chega a justificar o desuso dos equipamentos devido serem considerados desconfortáveis e capazes de reduzirem a produtividade ${ }^{17,18}$. Entretanto, muitos trabalhadores rurais e urbanos tem resguardado sua saúde mediante à práticas de higiene pessoal após a jornada de trabalho ${ }^{19}$.

No estudo de Farred et al. ${ }^{7}$ hábitos de higiene pessoal (lavagem das mãos, banho e troca da roupa) ao final do dia de trabalho foram adotadas por todas as mulheres avaliadas. Da mesma forma, $98 \%$ dos trabalhadores, lavam as mãos e 77\% tomam banho após o trabalho; além disso, 77\% deles lavam suas vestimentas de trabalho em separado das demais roupas, alegando medo de contaminação e forte mal cheiro que impregna naquelas ${ }^{3}$.

De maneira unânime os trabalhadores avaliados por este estudo alegaram, mediante as entrevistas individuais, que a higiene pessoal é uma prática rotineira após a jornada de trabalho. Além disso, foi possível perceber que os indivíduos que relataram exacerbação de sintomas respiratórios também concordavam que esses sintomas reduziam sua intensidade ou desapareciam logo após a auto-higienização.

A dificuldade de acesso à saúde é um marco na população investigada. Esse entrave é facilmente percebido mediante as avaliações e entrevistas individuais. Através desses objetos semiológicos, notou-se que parcela significativa dos voluntários nunca tinha aferido a pressão arterial, apesar de esse procedimento ser bastante simples, objetivo e acima de tudo, básico.

Acanhados, tímidos e reservados: esse foi o perfil observado na maioria dos trabalhadores rurais bujaruenses voluntários desta pesquisa. Segundo os dados obtidos na anamnese, contatou-se, direta ou indiretamente, que o trabalhador em questão possui baixo nível de instrução, situação sócio-econômica desvantajosa, e grande dificuldade em ter acesso aos serviços de saúde. Além disso, o adoecimento não costuma ser alvo da preocupação desses trabalhadores, ao contrário: dores e desconfortos, geralmente, são contornados com práticas de medicina popular.

Outro mecanismo de defesa desenvolvido pelos agricultores deste estudo equivaleu a adoção de práticas empíricas, conhecimento transpassado de pai para filho e experiências individuais. Através das entrevistas 
individuais, foi possível detectar que os aplicadores de agrotóxicos evitavam executar suas atividades sob condições climáticas adversas (sol e/ou vento excessivos). Ao serem questionados sobre esse saber, apenas disseram que há muitos anos tem-se esse hábito, já que o mesmo contribui para reduzir a freqüência dos sintomas.

Ressalta-se, entretanto, que os agricultores que não utilizavam EPI em suas atividades laborais apresentaram um aumento do tempo expiratório (TFEF) significando ser esse um índice preditivo espirométrico à disfunção obstrutiva (Tabela 2). Tal fato chama a atenção para a possibilidade de evoluir-se à obstrução do fluxo aéreo mais evidente, em detrimento dos indivíduos usuários dos equipamentos de proteção.

Dos avaliados, 65\% eram tabagistas ou ex-tabagistas de longa data, enquanto 35\% eram não tabagistas. Ao contrário do que se esperou, a variável que apresentou real associação com o tabagismo foi a CVF. Apenas essa variável elucidou resultado estatisticamente significante ( $p$-valor $=0.0037$ ), mostrando que, nos trabalhadores fumantes ou ex-fumantes, é mais acentuada a diferença de valores de CVF (L) obtidos em relação aos preditos (Tabela 3). Conclui-se que entre os Fumantes (Média -0.5) e os Não-Fumantes (média $=0.00$ ) existe uma real diferença da variável CVF $(\mathrm{L})$.

Em análise feita com pecuaristas expostos a partículas de origem animal, foi observado que ex-tabagistas e não tabagistas apresentaram mais sibilância do que os fumantes ativos. Os autores discutem que os fumantes desenvolveram a doença da via aérea reativa - em outras palavras: a tolerância à fumaça do cigarro estaria contribuindo para que o agricultor tolerasse também os efeitos da inalação de partículas aéreas nocivas do ambiente ocupacional. Em síntese, os trabalhadores tabagistas desenvolveram resistência às agressões aéreas, um "efeito fumante saudável"21.

O achado supracitado ajudaria a compreender a ausência de declínios espirométricos significativos no agricultor fumante bujaruense. Entretanto, é importante frisar que Senhorinho et al. ${ }^{19}$ constataram, em suas análises, que há manifestação predominante de DVO nos indivíduos fumantes, caracterizando, desta forma, o surgimento de doença pulmonar obstrutiva crônica nestes trabalhadores, com prejuízo de todas as variáveis espirométricas relacionadas ao fluxo expiratório. Contudo, os achados da presente pesquisa demonstraram diferenças semelhantes entre todas as variáveis espirométricas (com exceção da CVF), tanto para fumantes e ex-fumantes, quanto para não fumantes.

O tempo de exposição refletiu-se diretamente sobre as alterações de FEF25\%. Dos avaliados, 61,5\% lidavam com agrotóxicos há menos de 10 anos, enquanto que 38,5\% trabalhavam com a aplicação de pesticidas há mais de 10 anos. Somente o FEF25\% apresentou resultado estatisticamente significante ( $\mathrm{p}$-valor $=0.0247$ ), tornando evidente que, nos trabalhadores com mais de 10 anos de exposição, é mais acentuada a diferença entre o FEF25\% (L/seg) obtido em relação ao esperado. (Tabela 4). Conclui-se que, entre os indivíduos Com Menos de 10 Anos de Exposição (Média = -0.03) e os Com Mais de 10 Anos de Exposição (média = -0.51), existe uma real diferença da variável FEF25\% (L/seg), ao comparar-se valores preditos e obtidos.

Quanto ao tempo de exposição, Zuskin et al. ${ }^{13}$ observaram redução em todas as variáveis espirométricas dos trabalhadores do sexo masculino, enquanto que, nas mulheres, houve perda significativa do FEF25\% diretamente proporcional ao tempo de trabalho com os agrotóxicos, corroborando com os resultados do presente estudo e destacando que a redução desse parâmetro espirométrico sugere obstrução mais evidente nas vias aéreas de grosso calibre.

Vale lembrar que a exposição prolongada a baixos níveis de antígenos orgânicos e/ou químicos inalados pode desencadear respostas inflamatórias sutis nos pulmões, que podem não produzir sintomas durante vários anos. As pneumonites crônicas por hiperssensibilização - como são chamadas - geram quadro inflamatório crônico pulmonar, que progride lentamente, com cicatrização parenquimatosa. Diante do exposto, o processo inflamatório poderia justificar a redução do PFE observada nos trabalhadores desta pesquisa, assim como o processo cicatricial progressivo dos pulmões justificaria as reduções de CVF e VEF1 ${ }^{21}$.

Uma questão interessante é que essa doença é mais comum em não-tabagistas do que em tabagistas. Tal afirmativa é relevante, pois confirma a teoria do "efeito fumante saudável" apresentada por Hoppin et al. ${ }^{22}$

\section{Conclusão}

A partir dos resultados da pesquisa, ficou evidente que o trabalho agrícola relacionado à aplicação de agrotóxicos contribui negativamente para alterações na função pulmonar. A espirometria revelou que os trabalhadores rurais avaliados apresentaram todas as variáveis abaixo do previsto, de acordo com o peso, o sexo e a idade. 
Do ponto de vista funcional, $11,8 \%$ dos indivíduos apresentaram resultados espirométricos sugestivos de distúrbio ventilatório restritivo leve a moderado. Os declínios observados na prova de função pulmonar dos demais trabalhadores demonstraram que há predisposição ao desenvolvimento de distúrbios restritivos (42,3\% dos indivíduos) e mistos (46,2\% dos indivíduos) da ventilação.

A presença de sintomatologia, o tabagismo, a não utilização de EPI e o prolongado tempo de exposição não se relacionaram com o prejuízo adicional à função pulmonar. Todos os trabalhadores apresentaram reduções gerais na espirometria, entretanto o hábito tabagístico potencializou a redução de CVF; a não utilização dos EPI comprometeu de forma mais evidente o TFEF; e o longo tempo de exposição se refletiu diretamente sobre as reduções de FEF25\%.

Novas análises, com amostragem mais expressiva e controle de variáveis relacionadas à co-morbidades serão úteis para aumentar o conhecimento científico sobre a saúde respiratória da população rural e consequentemente melhorar a assistência em saúde dessa parcela da sociedade.

\section{Referências}

1. Carneiro FF, Augusto LG. Dossiê ABRASCO: um alerta sobre os impactos dos agrotóxicos na saúde. Rio de Janeiro: EPSJV; 2015.

2. Mamane A, Baldi I, Tessier JF, Raherison C, Bouvier G. Occupational exposure to pesticides and respiratory health. Eur Respir Rev. 2015;24(136):306-19. http://dx.doi.org/10.1183/16000617.00006014. PMid:26028642.

3. Rigotto RM, Vasconcelos DP, Rocha MM. Pesticide use in Brazil and problems for public health. Cad Saud Pub. 2014;30(7):1360-2. http:// dx.doi.org/10.1590/0102-311XPE020714. PMid:25166932.

4. Bendetti D. An evaluation of occupational exposures to pesticides in Brazil. Occup Med Health Aff. 2014;2(04):170. http://dx.doi. org/10.4172/2329-6879.1000170.

5. Nerilo SB, Martins FA, Nerilo LB, Salvadego VEC, Endo RY, Rocha GHO, et al. Pesticide use and cholinesterase inhibition in small-scale agricultural workers in southern Brazil. Braz J Pharm Sci. 2014;50(4):783-91. http://dx.doi.org/10.1590/S1984-82502014000400014.

6. Alif SM, Dharmage SC, Benke G, Dennekamp M, Burgess JA, Perret JL, et al. Occupational exposure to pesticides are associated with fixed airflow obstruction in middle-age. Thorax. 2017;72(11):990-7. http://dx.doi.org/10.1136/thoraxjnl-2016-209665. PMid:28687678.

7. Fareed M, Pathak MK, Bihari V, Kamal R, Srivastava AK, Kesavachandran CN. Adverse respiratory health and hematological alterations among agricultural workers occupationally exposed to organophosphate pesticides: A cross-sectional study in North India. PLoS One. 2013;8(7):697-701. http://dx.doi.org/10.1371/journal.pone.0069755. PMid:23936093.

8. Hernández AF, Casado I, Pena G, Gil F, Villanueva E, Pla A. Low level of exposure to pesticides leads to lung dysfunction in occupationally exposed subjects. Inhal Toxicol. 2008;20(9):839-49. http://dx.doi.org/10.1080/08958370801905524. PMid:18645724.

9. Shama FA, Skogstad M, Nijem K, Bjertness E, Kristensen P. Cross-shift changes in lung function among palestinian farmers during high- and low-exposure periods to pesticides: A longitudinal study. Arch Environ Occup Health. 2015;70(4):218-24. http://dx.doi.org/10.1080/193 38244.2013.859122. PMid:25386741.

10. De Jong K, Boezen HM, Kromhout H, Vermeulen R, Postma DS, Vonk JM. Association of occupational pesticide exposure with accelerated longitudinal decline in lung function. Am J Epidemiol. 2014;179(11):1323-30. http://dx.doi.org/10.1093/aje/kwu053. PMid:24780843.

11. Pereira CAC, Lemle A, Algranti E, Jansen JM, Valença LM, Nery LE. I Consenso Brasileiro sobre Espirometria. J Pneumol. 1996;22(3):105-64.

12. Ayres M, Ayres JR, Ayres DL, Santos AAS. BioEstat 5: aplicações estatísticas nas áreas das ciências biológicas e médicas. 5ed. Belém: Publicações Avulsas do Mamirauá; 2007.

13. Zuskin E, Mustajbegovic J, Schachter EN, Kern J, Deckovic-Vukres V, Trosic I, et al. Respiratory function in pesticide workers. J Occup Environ Med. 2008;50(11):1299-305. http://dx.doi.org/10.1097/JOM.0b013e3181845f6c. PMid:19001956.

14. Chakraborty S, Mukherjee S, Roychoudhury S, Siddique S, Lahiri T, Ray MR. Chronic exposures to cholinesterase-inhibiting pesticides adversely affect respiratory health of agricultural workers in India. J Occup Health. 2009;51(6):488-97. http://dx.doi.org/10.1539/joh. L9070. PMid:19851039.

15. Salameh P, Waked M, Baldi I, Brochard P, Saleh BA. Respiratory diseases and pesticide exposure: A case-control study in Lebanon.J Epidemiol Community Health. 2006;60(3):256-61. http://dx.doi.org/10.1136/jech.2005.039677. PMid:16476757.

16. Salameh P, Waked M, Baldi I, Brochard P. Spirometric changes following the use of pesticides. East Mediterr Health J. 2005;11(1-2):126-36. PMid:16532681.

17. Faria NMX, Facchini LA, Fassa AG, Tomasi E. Pesticides and respiratory symptoms among farmers. Rev Saú Públ. 2005;39(6):973-81. http:// dx.doi.org/10.1590/S0034-89102005000600016. PMid:16341409. 
18. Alves de Souza N, Inácia de Souza A, Bastos C, Rocha J, Oliveira C, Costa da Silva V. Prevalecência de distúrbios respiratórios associados ao uso de agrotóxicos em trabalhadores rurais em uma cidade da Zona da Mata Mineira. Rev Insp. 2010;2:6-10.

19. Senhorinho HC., Maria S, Gomes M, Franqui E, Júnior HP. Prevalência de distúrbios ventilatórios em trabalhadores rurais expostos a defensivos químicos no norte do Paraná. Fisio e Pesq. 2005;12(2):35-44. https://doi.org/10.1590/fpusp.v12i2.76502.

20. Schulte P, Geraci C, Zumwalde R, Hoover M, Kuempel E. Occupational risk management of engineered nanoparticles. J Occup Environ Hyg. 2008;5(4):239-49. http://dx.doi.org/10.1080/15459620801907840. PMid:18260001.

21. Scanlan CL, Wilkins RL, Stoller JK. Fundamentos da terapia respiratória de Egan. 7. ed. São Paulo: Manole; 2000

22. Hoppin JA, Umbach DM, London SJ, Alavanja MCR, Sandler DP. Animal production and wheeze in the Agricultural Health Study: interactions with atopy, asthma, and smoking. Occup Environ Med. 2003;60(8):e3. http://dx.doi.org/10.1136/oem.60.8.e3. PMid:12883030.

\section{Autor correspondente}

Samires Avelino de Souza França

Universidade Federal do Pará - UFPA

Av. Rodolfo Chermont, Pass. São Tomé, 995

CEP 66620-600, Marambaia, Belém, PA, Brasil

Tel.: (91) 98873-9093

E-mail:valeriafisio@gmail.com

\section{Informação sobre os autores}

SASF é fisioterapeuta mestre em Biologia de Agentes Infecciosos e Parasitários pela Universidade Federal do Pará.

PESA é fisioterapeuta doutor em Biotecnologia pela Universidade Federal do Pará, docente da Universidade da Amazônia.

VMFN é fisioterapeuta doutora em Neurociências e Biologia Celular pela Universidade Federal do Pará, docente da Universidade do Estado do Pará.

\section{Contribuição dos autores}

SASF - coleta dos dados; PESA - análise dos dados; VMFN - orientador. 